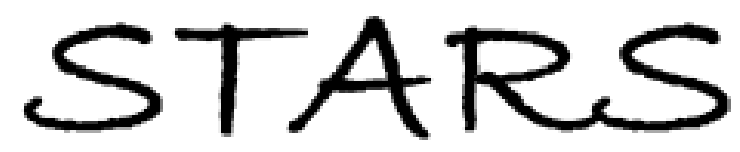

University of Central Florida

STARS

$1-1-2013$

\title{
An apparatus for studying scintillator properties at high isostatic pressures
}

\author{
R. M. Gaumé
}

S. Lam

University of Central Florida

M. Gascón

University of Central Florida

W. Setyawan

S. Curtarolo

See next page for additional authors

Find similar works at: https://stars.library.ucf.edu/facultybib2010

University of Central Florida Libraries http://library.ucf.edu

This Article is brought to you for free and open access by the Faculty Bibliography at STARS. It has been accepted for inclusion in Faculty Bibliography 2010 s by an authorized administrator of STARS. For more information, please contactSTARS@ucf.edu.

\section{Recommended Citation}

Gaumé, R. M.; Lam, S.; Gascón, M.; Setyawan, W.; Curtarolo, S.; and Feigelson, R. S., "An apparatus for studying scintillator properties at high isostatic pressures" (2013). Faculty Bibliography 2010s. 4007. https://stars.library.ucf.edu/facultybib2010/4007

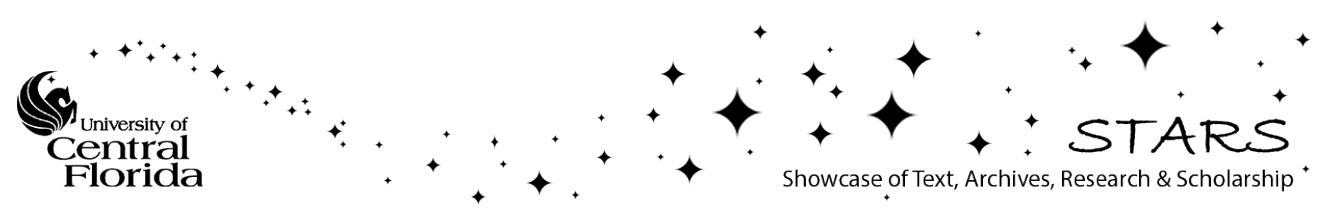




\section{Authors}

R. M. Gaumé, S. Lam, M. Gascón, W. Setyawan, S. Curtarolo, and R. S. Feigelson 


\title{
An apparatus for studying scintillator properties at high isostatic pressures
}

\author{
R. M. Gaumé, ${ }^{1, a)}$ S. Lam, ${ }^{2}$ M. Gascón, ${ }^{2}$ W. Setyawan, ${ }^{3}$ S. Curtarolo, ${ }^{4}$ and R. S. Feigelson ${ }^{2}$ \\ ${ }^{1}$ College of Optics and Photonics (CREOL) and NanoScience Technology Center, University of Central \\ Florida, Orlando, Florida 32816, USA \\ ${ }^{2}$ Department of Materials Science and Engineering, Stanford University, Stanford, California 94305, USA \\ ${ }^{3}$ Pacific Northwest National Laboratory, Richland, Washington 99352, USA \\ ${ }^{4}$ Department of Mechanical Engineering and Materials Science, Duke University, Durham, North Carolina \\ 27708, USA
}

(Received 1 September 2012; accepted 13 December 2012; published online 9 January 2013)

\begin{abstract}
We describe the design and operation of a unique hydraulic press for the study of scintillator materials under isostatic pressure. This press, capable of developing a pressure of a gigapascal, consists of a large sample chamber pressurized by a two-stage hydraulic amplifier. The optical detection of the scintillation light emitted by the sample is performed, through a large aperture optical port, by a photodetector located outside the pressure vessel. In addition to providing essential pressuredependent studies on the emission characteristics of radioluminescent materials, this apparatus is being developed to elucidate the mechanisms behind the recently observed dependency of light-yield nonproportionality on electronic band structure. The variation of the light output of a Tl:CsI crystal under 511-keV gamma excitation and hydrostatic pressure is given as an example. () 2013 American Institute of Physics. [http://dx.doi.org/10.1063/1.4773563]
\end{abstract}

\section{INTRODUCTION}

Scintillator materials have long been used in the detection of ionizing radiation and remain to this day at the core of a variety of detectors for medical diagnostics, geological prospecting, particle physics, and homeland security. The performance of these materials, which luminesce when exposed to high-energy radiation, depends on application-specific parameters including the light output, emission wavelength, and decay time. For example, applications requiring the discrimination of incoming $\gamma$-rays at different energies necessitate high light yields for improving photon-counting statistics and a good linearity between the deposited energy and the number of light-photons produced per event. ${ }^{1}$ In response to specific needs posed by nuclear surveillance applications, the quest for understanding and finding scintillator materials with superior $\gamma$-ray energy resolution and light-yield proportionality that would approach the performance of costly semiconductor-based detectors has been intensified during the last decade..$^{2-8,20}$

In this context, recent computational modeling by Setyawan et $a .^{8}{ }^{8}$ identified a relationship between the effective mass ratio of the charge carriers produced in the ionization track and the scintillator's light-yield proportionality. This study also suggested that the application of large isostatic pressures could affect scintillator electronic band structure and lead to important insight into this relationship. It is known that pressure affects the migration and recombination properties of free charge carriers and excitons (through self-trapping, change in exciton binding energies, ${ }^{9,10}$ hopping and stability of states ${ }^{11-18}$ ) and, as a result, impacts

\footnotetext{
a) This research was performed while R. Gaumé was at the Department of Materials Science and Engineering, Stanford University, Stanford, California 94305 , USA. Author to whom correspondence should be addressed. Electronic mail: gaume@ucf.edu.
}

the deposited energy to photon-yield ratio as confirmed by a few high isostatic pressure scintillation studies. For example, $30 \mathrm{MPa}$ of isostatic pressure induces a change in the light yield and energy response of Tl:NaI excited by $\gamma$ rays. ${ }^{19}$ Gamma-ray scintillation measurements in the $\mathrm{keV}-\mathrm{MeV}$ energy range require scintillator sample sizes on the order of the material's stopping power (from a few millimeters to a couple of centimeters) to provide sufficient interaction volume. ${ }^{20}$ For this reason, the specific study motivated here cannot make use of small volume diamond anvil cells and necessitates a high pressure system capable of handling centimeter-size samples in a vessel equipped with an optically-transmissive port for the collection of scintillation light. To minimize the risk associated with the handling of compressed gases, the system is designed to utilize pressure-transmitting liquids. The press described in this paper was built by Harwood Engineering, Inc. (Walpole, MA) and Secs. II-IV describe the various components and characteristics of the completed apparatus, as well as some preliminary scintillation measurements.

\section{SYSTEM COMPONENTS AND OPERATION}

\section{A. Pressure vessel}

The pressure vessel at the core of this instrument consists of a $21-\mathrm{cm}$ diameter cylinder made of 4340-stainless steel with a sample chamber accessible by a top-loading threaded steel plug. The plug is equipped with an electrical feed-through for monitoring temperature changes inside the chamber with a type $\mathrm{K}$ thermocouple. The sample chamber contains both the centimeter-sized scintillator and a hollow cylindrical spacer holding the solid-state radioactive source (e.g., electro-deposited $\gamma$-ray radionuclide standard source) at a fixed distance from the sample. The sample sits directly on the optical window located at the base of the vessel and the remaining volume is filled with a high-pressure 

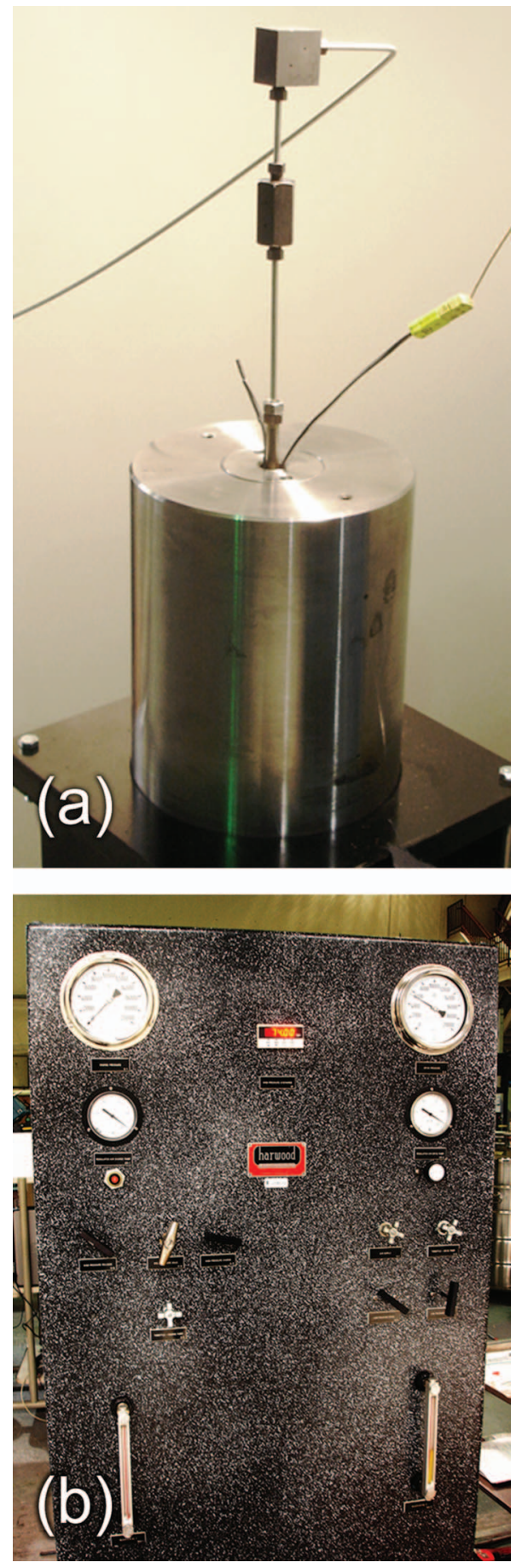

FIG. 1. The pressure vessel (a) and control panel (b).

transmitting fluid. Contrary to typical conditions used for the characterization of scintillators under ambient pressure in air, the presence of the pressure-transmitting fluid and the application of pressure are not compatible with the use of optical coupling silicone grease (index of typical silicone grease $n_{D}$ $=1.465$ ). We have found that the crystallization of the grease under pressure deteriorates the light collection. As a result, the scintillator is simply wrapped in an enhanced specular reflective (ESR) film (such as Vikuiti by $3 \mathrm{M}^{\mathrm{TM}}$ ) leaving only one face free for coupling to the optical window. The free area of the window's face and the sides are also covered with

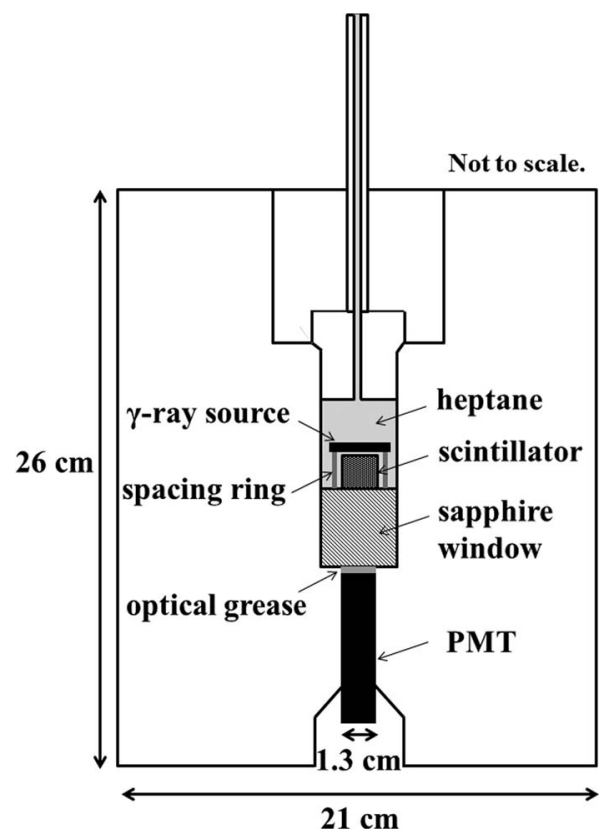

FIG. 2. Schematic of the pressure vessel with the scintillator, radioactive source and photomultiplier tube (PMT).

ESR to maximize light collection by the photodetector. The reflective properties of the ESR film vary reversibly with the application of pressure, up to at least $800 \mathrm{MPa}$, and for a few pressurization cycles. It was noted however, that, after half a dozen cycles, the film develops visible striations and irreversible damage, likely due to excessive differential stresses in this laminated film.

On the outside, the optical window can be coupled to a photomultiplier tube (PMT), an avalanche photodiode or a bundle of optical fibers. Pulse-height $\gamma$-ray spectra and decay curves are collected by directing the photodetector signal output to a chain of standard pulse-processing electronics (i.e., amplifier and multichannel analyzer) ${ }^{21}$ or to an oscilloscope, respectively. The optical fiber is used with a spectrometer to obtain emission spectra. The pressure vessel and the control panel as well as a schematic of the vessel are shown in Figs. 1 and 2. A summary of the specifications for the vessel is given in Table I.

TABLE I. Pressure vessel specifications.

\begin{tabular}{lc}
\hline \hline Component & Specification \\
\hline Pressure range & $0-1 \mathrm{GPa}$ \\
Working temperature & $(20 \pm 10)^{\circ} \mathrm{C}$ \\
Pressurizing fluid & Heptane \\
Vessel material & E-4340 AQ steel (heat-treated and \\
& auto-frettagged) \\
Vessel dimensions & $\varnothing 21.0 \mathrm{~cm} \times 29.0 \mathrm{~cm}$ \\
Sample chamber & $\varnothing 3.5 \mathrm{~cm} \times 4.1 \mathrm{~cm}$ long \\
Optical window & $\varnothing 3.0 \mathrm{~cm} \times 3.2 \mathrm{~cm}$ long, c-oriented \\
& sapphire crystal \\
Electrical lead-throughs & Zirconium-brass alloy \\
\hline \hline
\end{tabular}


TABLE II. Mechanical properties of high-strength window materials.

\begin{tabular}{lcccc}
\hline \hline & Flexural strength (MPa) & Bulk modulus (GPa) & Young modulus (GPa) & Shear modulus (GPa) \\
\hline Sapphire & $500-1000$ & 250 & 345 & 145 \\
Spinel & 170 & 197 & 276 & 109 \\
YAG & $280-530$ & 221 & 290 & 115 \\
AlON & 379 & 210 & 323 & 125 \\
\hline \hline
\end{tabular}

\section{B. Sapphire window}

The optical window material selection and design were guided by the stringent requirements on this apparatus: (1) the window must be capable of withstanding a GPa of flexural stress, (2) it should have a large aperture to permit the light collection from centimeter-sized scintillators, and (3) it must be transparent over the visible spectral range $(300-600 \mathrm{~nm})$ to accommodate a variety of scintillator emissions. Despite the potential optical losses due to the birefringent nature $\left(\mathrm{n}_{\mathrm{o}}\right.$ $=1.768, \mathrm{n}_{\mathrm{e}}=1.760$ ) and the photo-elastic properties of sapphire, this material was preferred over cubic spinel $\mathrm{MgAl}_{2} \mathrm{O}_{4}$, YAG, or AlON because of its superior flexural strength ${ }^{22-24}$ (Table II), availability and lower cost.

Cylindrical sapphire windows, $3.0 \mathrm{~cm}$ in diameter, $3.2 \mathrm{~cm}$ thick, and oriented along the c-axis, were obtained from GTAT Crystal Systems, LLC (Salem, MA). Initially, the windows were given an optical polish on both ends and a rough polish on the outer periphery. ${ }^{25}$ The windows showed no measurable built-in stress under crossed-polarizers. However, tests performed by Harwood Engineering, Inc. showed that these windows were unable to withstand the shearing forces that developed near the bottom seat of the window and critical failure occurred at about $0.8 \mathrm{GPa}$. The failure modes ranged from diagonally-oriented holes "drilled" in the bulk of the window to catastrophic fragmentation into sapphire "sand." Fracture in c-oriented, uniaxially-loaded, single-crystalline sapphire proceeds along the c-axis and occurs when crystal twins on differently-oriented rhombohedral crystal planes (r-planes) intersect with each other ${ }^{26}$ Compression on the c-axis produces equally-resolved shear stresses on all the r-planes, ${ }^{26}$ and the stress required to form twins on one r-plane induces twins on the two other intersecting planes. Atoms at these intersections are then sheared in one direction by one twin and in a different direction by the other twin, leading to crack initiation. The brittle behavior observed in the early version of the sapphire windows was induced by crack propagation from the ground surfaces and sharp edges. Sapphire windows with $20-10$ polish of the flat surfaces, fine grind on the outer diameter and appropriate chamfering of the edges to $\sim 0.015$ in. $\times 45^{\circ}$ showed significant strength improvement and were capable of withstanding a GPa for more than 50 loading cycles.

\section{Hydraulic components and operation}

The pressurization system (see schematic in Fig. 3) consists of a dual-stage hydraulic compressor. The circuit represented in the upper portion of Fig. 3 first pressurizes the sample chamber to the maximum pressure that the air-driven pump (P1) can develop (0.15 GPa for a 100-psi compressed air input) by forcing the high-pressure transmitting fluid from a storage tank (T1) to the pressure vessel. In this first stage of compression, the pressure intensifier's piston rests at the bottom of its chamber. Next, the activation of the second hydraulic circuit (lower part of Fig. 3) pressurizes the lowpressure hydraulic fluid from its tank (T2) to the pressureintensifier with the aid of the air-driven pump (P2). As a result, the piston of the pressure intensifier moves upward and increases the pressure in the vessel further. The system is equipped with three pressure gauges (G1, G2, and G3) to monitor the driving pressures as well as the pressure in the vessel. High-pressure needle valves (V1-V5), air regulators (RG1 and RG2), and air gauges (AG1 and AG2) assist the flow control of air entering the pumps and pressurizing fluids while filters (F, F1, and F2) ensure that small debris or particulates do not circulate in the system. A rupture disk prevents any unintended over-pressurization of the pressure vessel.

\section{Pressure-transmitting fluids}

As mentioned in Sec. II C, two pressure-transmitting fluids circulate in two independent circuits. A clean, filtered, and well-refined synthetic hydraulic oil (e.g., antiwear oil AW32) is used as the low-pressure transmitting fluid (reservoir T2). The selection of the high-pressure transmitting fluid (located in T1) presents more challenges since this fluid should be relatively incompressible in order to transmit pressure to the sample chamber and should not undergo crystallization under compression up to a GPa at ambient temperature. Furthermore, it has to be optically transparent, not fluorescent or radioluminescent under $\gamma$-excitation, and perfectly anhydrous since it is in direct contact with samples that may be hygroscopic (such as $\mathrm{NaI}, \mathrm{SrI}_{2}$, or $\mathrm{LaBr}_{3}$ ). Other considerations include low toxicity, good lubricating qualities, and a low flash-point. Heptane (Sigma-Aldrich, spectrophotometric grade, 99\%) was found to be a suitable fluid for this purpose. When immersed in the pressure transmitting fluid, the coupling between the ESR film and the sample improves slightly since heptane has a higher refraction index than air $\left(\mathrm{n}_{\mathrm{D}}=1.387\right.$ at $\left.1 \mathrm{~atm}\right)$.

\section{SYSTEM PERFORMANCE}

\section{A. Pressure and temperature stability}

All scintillation measurements must be conducted at constant pressure as well as constant temperature, particularly since temperature is known to affect scintillation properties. ${ }^{27-33}$ The temperature of the fluid in the sample 


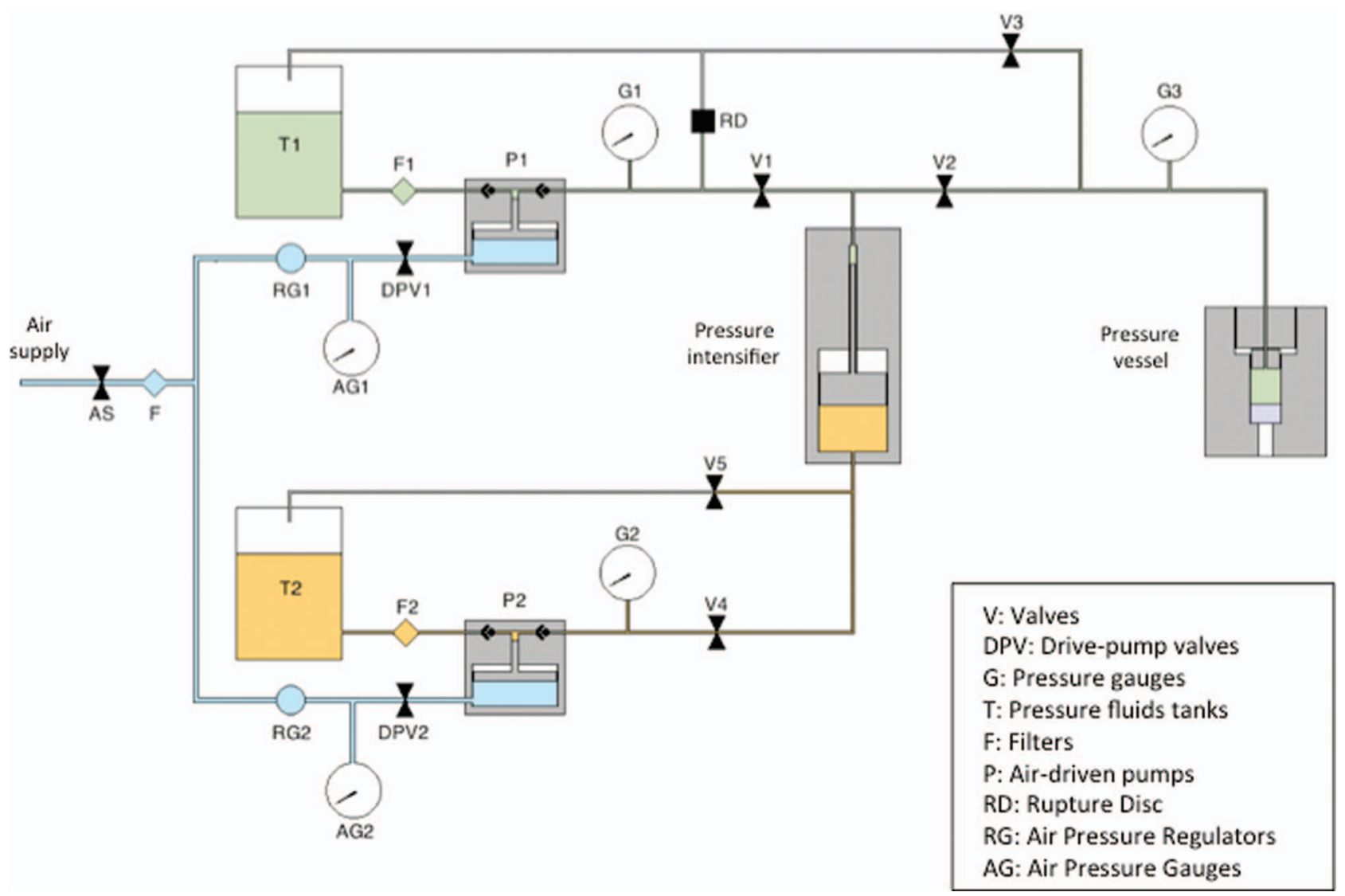

FIG. 3. Schematic of the hydraulic components driving the press.

chamber increases and decreases significantly due to adiabatic heating and cooling during the compression and decompression phases, respectively. This effect leads to temperature and density gradients in the fluid, which can induce non-uniform stresses on the scintillator sample and the window, and result in their fracture. Reducing the compression and decompression rates to about a GPa per 15 min can minimize such risks. Thermal stability must also be achieved before sample measurements can be made. Figure 4 illustrates the typical evolution of temperature inside the pressure vessel after compressing and isolating the vessel by closing valve V3. The heat generated by adiabatic compression is slowly dissipated through the bulk of the vessel and the temperature equilibrates to the ambient value. During normal operation, temperature stability is achieved within $5 \mathrm{~min}$.

The pressure stability of the system can be determined once thermal stability is achieved. Figure 5 shows the time evolution of pressure after an initial compression of $0.517 \mathrm{GPa}$. Over a period of $1 \mathrm{~h}$, the relative pressure drop was less than $1 \%$. Since a typical scintillation or emission measurement requires an acquisition time on the order of 10 min, the stability of the pressure system was determined to be satisfactory for our purposes.

\section{B. Pressure-dependent transmission}

The refractive indices of the scintillator, heptane, and, to a lesser extent, the sapphire optical window are each expected to change with pressure. Accordingly, the coupling and trans- mission behavior for these media are also expected to vary, which will affect the measured light output of the scintillator. To determine the nature of this dependency, the changes in the light transmission from the scintillator through the heptane and the sapphire window were characterized as a function of applied pressure up to $0.75 \mathrm{GPa}$ for a $1-\mathrm{cm}^{3} \mathrm{Tl}$ :CsI scintillator. A 35-W high-pressure xenon light source (Ocean Optics HPX 2000) was coupled to a reflection probe (Ocean Optics Reflection/Backscattering Probe, R600-7-UV/125F) and

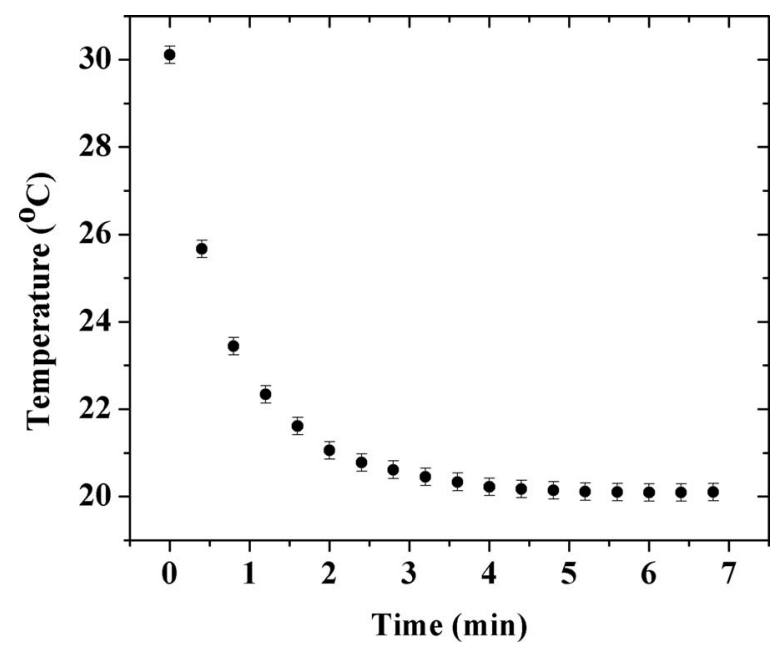

FIG. 4. Temperature equilibration inside the pressure chamber after compression. 


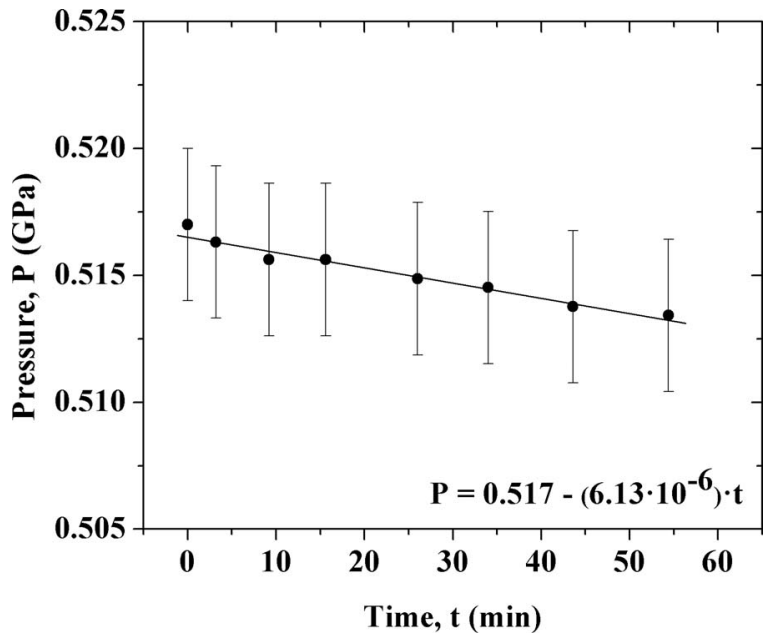

FIG. 5. Loss of pressure inside the vessel over time. After initial compression to $0.517 \mathrm{GPa}$ the vessel was isolated for $1 \mathrm{~h}$.

to the outer end of the sapphire window. The Tl:CsI sample was illuminated below bandgap at $550 \mathrm{~nm}$, a wavelength at which no luminescence is produced in the crystal, and the reflected light was collected by the reflection probe and analyzed by a spectrophotometer (Ocean Optics, QE65000). The pressure-dependent transmission behavior (normalized to its value at ambient pressure) varied up to $3.5 \%$ (Fig. 6). Note that a different transmission curve would be generated for each scintillator studied. These transmission curves allow for appropriate corrections to be made when determining the light output of a material.

\section{APPLICATION EXAMPLE: SCINTILLATION STUDY OF TI:CsI UNDER PRESSURE}

The pressure system was used to test a $1-\mathrm{cm}^{3}$ sample of Tl:CsI obtained from Hilger Crystals. The sample was polished on one face and wrapped in ESR film as described in the operation procedure. Pulse-height spectra (Fig. 7(a)) were recorded up to $0.7 \mathrm{GPa}$ for a $511-\mathrm{keV}$ solid excitation source, ${ }^{22} \mathrm{Na}$ (Eckert and Ziegler Isotopes). Despite the less than opti-

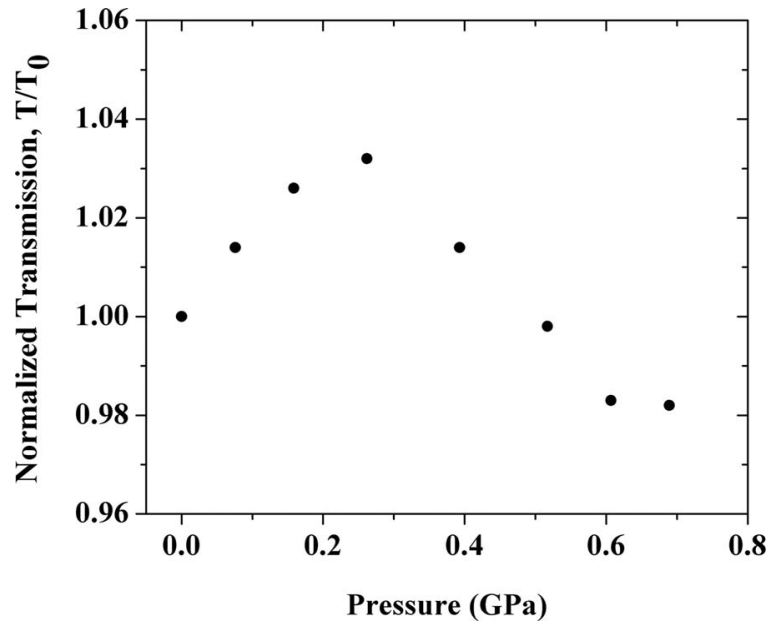

FIG. 6. Pressure-induced changes in the transmission of the optical arrangement with a $1-\mathrm{cm}^{3}$ Tl:CsI crystal.
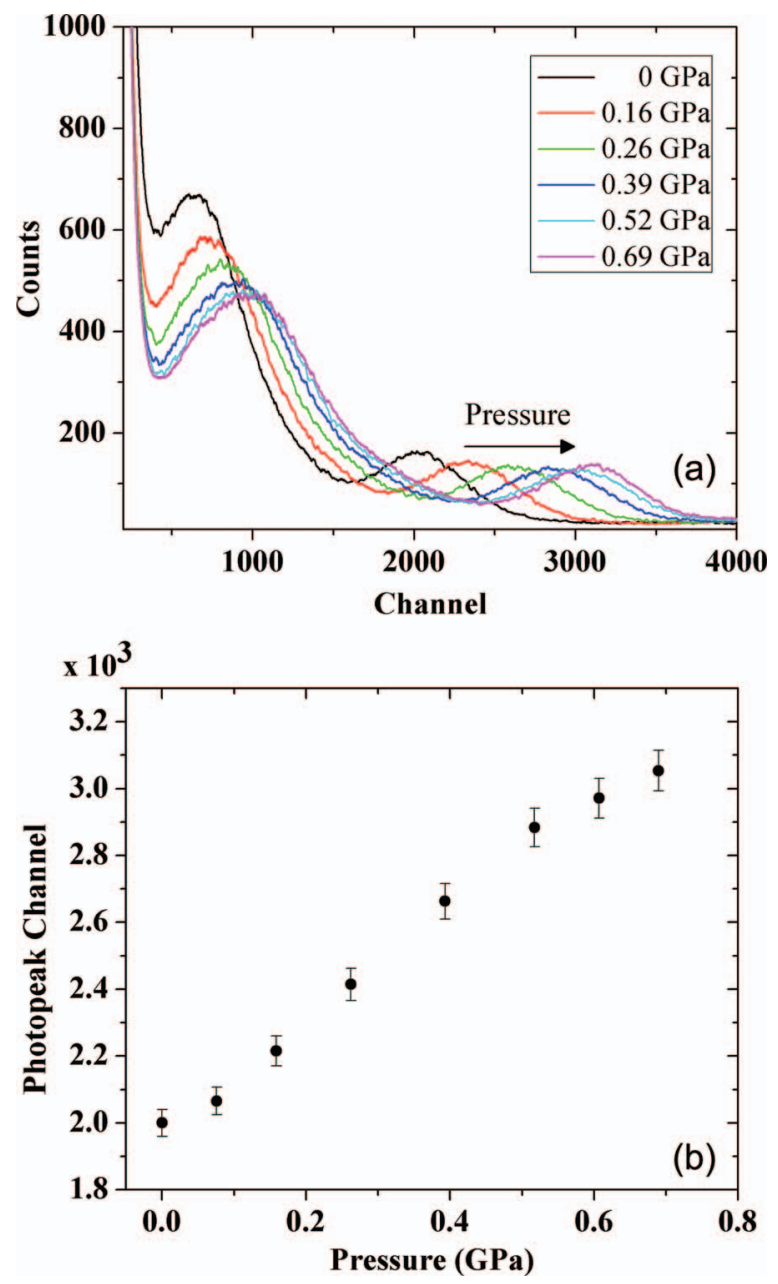

FIG. 7. (a) Pulse-height gamma-ray spectra of a $1-\mathrm{cm}^{3}$ Tl:CsI under 511$\mathrm{keV}\left({ }^{22} \mathrm{Na}\right)$ excitation as a function of pressure, and (b) shift of the photopeak to higher channels, indicating that light output increased at higher pressures. Error bars originates from the fitting procedure.

mal light coupling through the thick sapphire window, it was observed that the location of the photopeak, which is proportional to the light output, increases reversibly with increasing isostatic pressure (Fig. 7(b)). The photopeak centroid was determined by fitting the full-energy peaks in the pulse-height spectra using a Gaussian function and an exponential background. Reasons for the observed trend are currently under investigation.

\section{SUMMARY}

A high isostatic pressure system consisting of a pressure vessel fitted with an optical port and pressurized by a twostage hydraulic compressor was designed and constructed for the study of centimeter-sized inorganic scintillators up to $1 \mathrm{GPa}$. The system performance was found suitable for typical scintillation measurements. Tests on a Tl:CsI scintillator under $511-\mathrm{keV}$ excitation up to $0.7 \mathrm{GPa}$ were successfully performed and a dependency between the light yield and the applied isostatic stress was observed. We anticipate that this press will be extremely useful in investigating a variety of scintillation properties (light yield, decay 
time, energy resolution, and light yield nonproportionality) under high isostatic pressure and allow a better understanding of how those properties relate to the crystalline structure of scintillators.

\section{ACKNOWLEDGMENTS}

The authors wish to thank W. Newhall of Harwood Engineering, Inc. and GTAT Crystal Systems, LLC for their helpful discussions. This work was supported by the Domestic Nuclear Defense Office (DNDO) under Grant No. 2008-DN077-ARI005-02.

${ }^{1}$ W. Mengesha, T. D. Taulbee, B. D. Rooney, and J. D. Valentine, IEEE Trans. Nucl. Sci. 45, 456 (1998).

${ }^{2}$ P. Dorenbos, J. T. M. de Haas, and C. W. E. van Eijk, IEEE Trans. Nucl. Sci. 42, 2190 (1995).

${ }^{3}$ T. D. Taulbee, B. D. Rooney, W. Mengesha, and J. D. Valentine, IEEE Trans. Nucl. Sci. 44, 489 (1997).

${ }^{4}$ W. W. Moses, S. A. Payne, W.-S. Choong, G. Hull, B. W. Reutter, IEEE Trans. Nucl. Sci. 55(3), 1049 (2008).

${ }^{5}$ S. A. Payne, N. J. Cherepy, G. Hull, J. D. Valentine, W. W. Moses, and W.-S. Choong, IEEE Trans. Nucl. Sci. 56(4), 2506 (2009).

${ }^{6}$ S. A. Payne W. W. Moses, S. Sheets, L. Ahle, N. J. Cherepy, B. Sturm, S. Dazeley, G. Bizarri, and W.-S. Choong, IEEE Trans. Nucl. Sci. 58(6), 3392 (2011).

${ }^{7}$ M. Moszyński, Radiat. Meas. 45(3-6), 372 (2010).

${ }^{8}$ W. Setyawan, R. M. Gaume, R. S. Feigelson, and S. Curtarolo, IEEE Trans. Nucl. Sci. 56, 2989 (2009).

${ }^{9}$ R. S. Knox, Theory of Excitons (Academic, New York, 1963).

${ }^{10}$ E. O. Kane, J. Phys. Chem. Solids 1, 249 (1957).

${ }^{11}$ T. Tsujimoto, H. Nishimura, and M. Nakayama, Phys. Rev. B 54, 16579 (1996).

${ }^{12}$ H. Nishimura, K. Kitano, S. Kawase, and M. Nakayama, Phys. Rev. B 57, 2592 (1998).
${ }^{13}$ H. Nishimura, T. Tsujimoto, M. Nakayama, T. Horiguchi, and M. Kobayashi, J. Phys. Soc. Jpn. 63, 2818 (1994).

${ }^{14}$ H. Nishimura, T. Tsujimoto, M. Nakayama, S. Morita, and M. Kobayashi, J. Lumin. 62, 41 (1994).

${ }^{15}$ T. Tsujimoto, H. Nishimura, M. Nakayama, H. Kurisu, and T. Komatsu, J. Lumin. 60/61, 798 (1994)

${ }^{16} \mathrm{H}$. Nishimura, T. Tsujimoto, S. Morimoto, and M. Nakayama, J. Lumin. 58, 347-349 (1994)

${ }^{17}$ V. Babin, J. Lumin. 76, 502 (1998).

${ }^{18}$ S. Zazubovich, Radiat. Meas. 33, 699 (2001).

${ }^{19}$ R. Hill and A. J. L. Collinson, Br. J. Appl. Phys. 17, 1377 (1966).

${ }^{20}$ W. Setyawan, R. M. Gaume, S. Lam, R. S. Feigelson, and S. Curtarolo, ACS Comb. Sci. 13, 382 (2011).

${ }^{21}$ G. F. Knoll, Radiation Detection and Measurement, 4th ed. (Wiley, Hoboken, NJ, 2010), p. 596.

${ }^{22}$ J. C. Chervin, G. Syfosse, and J. M. Besson, Rev. Sci. Instrum. 65, 2719 (1994).

${ }^{23}$ E. R. Dobrovinskaya, L. A. Lytvynov, and V. Pischik, Sapphire: Material, Manufacturing, Applications (Springer, New York, NY, 2009).

${ }^{24}$ D. Harris, Report of the Naval Air Systems Command, China Lake, CA, May 2002, see http://www.dtic.mil/cgi-bin/GetTRDoc?AD=ADA421203

${ }^{25}$ A. H. Heuer, Philos. Mag. 13, 379 (1966).

${ }^{26} \mathrm{~F}$. Schmid and D. C. Harris, "Effect of crystal orientation and temperature on the strength of sapphire," J. Am. Ceram. Soc. 81, 885 (1998).

${ }^{27}$ P. A. Rodyni, Basic Principles and Processes in Physical Processes in Inorganic Scintillators (CRC/LLC, Boca Raton, FL, 1997), pp. 26-30.

${ }^{28}$ L. Swiderski, M. Moszynski, W. Czarnacki, A. Syntfeld-Kazuch, and M. Gierlik, IEEE Trans. Nucl. Sci. 54, 1372 (2007).

${ }^{29}$ M. Moszynski, A. Nassalski, A. Syntfeld-Kazuch, T. Szczesniak, W. Czarnacki, D. Wolski, G. Pausch, and J. Stein, Nucl. Instrum. Methods 568, 739 (2006)

${ }^{30}$ J. Gironnet, V. B. Mikhailik, H. Kraus, P. de Marcillac, and N. Coron, Nucl. Instrum. Methods Phys. Res. A 594, 358 (2008).

${ }^{31}$ E. Zych, C. Brecher, and J. Glodo, J. Phys.: Condens. Matter 12, 1947 (2000).

${ }^{32}$ S. Weber, D. Christ, M. Kurzeja, R. Engels, G. Kemmerling, and H. Halling, IEEE Trans. Nucl. Sci. 50, 1370 (2003).

${ }^{33}$ J. Glodo and A. J. Wojtowicz, J. Alloys Compd. 300, 289 (2000). 